\title{
INVESTIGACIONES
}

\section{ESTRATEGIAS DE APRENDIZAJE Y AUTOESTIMA. SU RELACION CON LA PERMANENCIA Y DESERCION UNIVERSITARIA*}

\author{
Learning strategies and self-esteem. Relationship between staying and dropping \\ out secondary school
}

\section{Olga María Fernández González, Macarena Martínez-Conde Beluzan, Roberto Melipillán Araneda}

\author{
Universidad Santo Tomás, Avda. Carlos Schorr 255, Talca. \\ mofernandez@santotomas.cl
}

\section{Resumen}

Este estudio tiene como objetivo describir las estrategias de aprendizaje y autoestima de los alumnos que ingresan a la Universidad Santo Tomás, sede Talca 2007, y determinar la relación existente entre ellas y el rendimiento académico. Para recolectar la información se utilizaron el Inventario de Estrategias de Aprendizaje de R. Schmeck (adaptado en Chile por Truffello y Pérez 1988) y el Inventario de Autoestima de Coopersmith forma A (adaptado en Chile por Brinkmann, Sègure y Solar 1989). Los resultados indican que los estudiantes universitarios que utilizan estrategias de aprendizaje más complejas presentan significativamente mejor rendimiento académico que los estudiantes que utilizan estrategias más simples, y además se caracterizan por presentar mayores niveles de autoestima general, de autoestima académica y autoestima familiar.

Palabras clave: educación superior, deserción, estrategias de aprendizaje, autoestima.

\begin{abstract}
This study describes the learning strategies and self-esteem of students from Santo Tomás University, campus Talca starting studies in 2007, and shows the relationship betweeen them and the academic results of these students. In order to recolect data it was used the Learning Strategies Inventory (R. Schmeck, adapted in Chile by Truffello \& Pérez 1988) and Coopersmith's Self-esteem Inventory Form A (adapted in Chile by Brinkmann, Segure \& Solar 1989). The results shows that students who use complex learning strategies have better academic results than those students who use simple strategies, thus the first group shows higher levels of general self-esteem, academic self-esteem, and familiar self-esteem.
\end{abstract}

Key words: secondary school, dropping out, learning strategies, self-esteem.

\footnotetext{
* Investigación financiada por Universidad Santo Tomás. Proyecto de Investigación, Creación y Desarrollo de la Docencia, 2007.
} 


\section{ANTECEDENTES TEORICOS Y EMPIRICOS}

La Educación Superior en muchos países se ha visto enfrentada a una serie de cambios que le definen un nuevo contexto. Por más de 500 años las universidades se desarrollaron a partir de modelos centrados en el desarrollo disciplinario, dirigido a una elite intelectual y académica, expresado en el encuentro cara a cara entre profesores y estudiantes, establecido éste desde el acceso a textos escritos. Como esta estructura se mantiene, pero el contexto ha cambiado, surge la incertidumbre acerca de la identidad de la Universidad y del rol que la sociedad le asigna al conocimiento, que ha adquirido una dimensión, un estatus y roles muy distintos de los tradicionales (González y Uribe 2002; Lemaitre 2003).

La incertidumbre se establece en función de factores como: la emergencia de un nuevo modo de generar conocimientos (enfocado en la necesidad de resolver problemas complejos); la globalización, los tratados internacionales comerciales y la difuminación de las fronteras, que obligan a certificar que los estándares educativos son similares a los internacionales, así como exige que se genere la capacidad de producir y aplicar conocimiento producido en otros países; y finalmente, el impacto producido por la masificación del acceso a la Educación Superior, que implica una población estudiantil más grande y más diversa (Lemaitre 2003).

Respecto del aumento de la cobertura en Educación Superior, cabe señalar que, aunque la cobertura en Chile es mayor que en el resto de los países de la región, aún es menor que en países desarrollados (EE.UU., Canadá), lo que implica que seguirá incrementándose en los próximos años, por lo que se hace necesario preocuparse de los problemas asociados a este aumento (González y Uribe 2002).

Una consecuencia del aumento de cobertura es la heterogeneidad de la población estudiantil. Ya no se trata de la elite, sino de un grupo, más grande, de características "normales", diverso en cuanto a sus conocimientos, habilidades y destrezas, y que muchas veces se encuentra con que la mayor parte de los programas universitarios han sido diseñados bajo el supuesto de una cierta homogeneidad, sin ajustar las modalidades docentes a los cambios (Himmel 2002; 2003).

El escenario se compone entonces de alumnos que inician estudios superiores sin contar con las competencias mínimas que requiere el programa de estudios, enfrentándose a dos situaciones posibles: algunos pueden persistir en sus metas a costa de múltiples repeticiones, prolongando la permanencia mucho más allá de la duración prevista, con la consiguiente frustración y costo económico. Otros, con menos tolerancia a la frustración o menos recursos económicos, terminan por desertar prematuramente. La situación es tal que, en promedio, la mitad de quienes ingresan a la Educación Superior abandonan antes de lograr el título o grado, siendo la mayor deserción durante el primer año (González y Uribe 2002; Himmel 2002; 2003).

Esta deserción tiene consecuencias sociales, personales y familiares, así como económicas, tanto a nivel individual como a nivel país (González y Uribe 2002; Himmel 2002; Montes 2002; Canales y De los Ríos 2007).

Con el fin de minimizar estas consecuencias es importante reconocer cuáles son los factores predictores de la deserción. A falta de un cúmulo importante de investigaciones al respecto en Chile, los modelos explicativos extranjeros dan cuenta de la importancia de factores tales como: las características individuales de los alumnos (motivación y 
vocación, creencias y actitudes, habilidades y destrezas cognitivas), características de la institución en la que estudian (tipos y calidad de los servicios que entregan) y factores sociales (las facilidades u obstáculos para que los alumnos se integren y comprometan con la institución) (Himmel 2002; Canales y De los Ríos 2007).

En este aspecto, los resultados de la más reciente investigación en Chile (Canales y De los Ríos 2007) corroboran en muchos sentidos los modelos explicativos ya mencionados. El análisis de las causas o factores explicativos de la deserción (temporal o permanente) muestra que este fenómeno se explica por un entramado de factores complejo que no puede ser fácilmente atribuido a una sola causa explicativa.

En este contexto, si las instituciones de Educación Superior no llevan a cabo un adecuado proceso de cambio que les permita acoger de mejor manera la diversidad estudiantil, pueden tender a rebajar los estándares, graduando profesionales que no alcanzan el perfil suficiente y necesario que definieron por carrera, con el consiguiente desprestigio de sus egresados y la dificultad de éstos para insertarse laboralmente. Por otro lado, si no disminuyen los estándares requeridos para el perfil profesional, se exponen a altas tasas de deserción, lo que tampoco las prestigia (Himmel 2003).

Una forma de superar los riesgos ya descritos es un acertado diagnóstico inicial, a fin de trabajar con aquellos factores individuales que afectan las tasas de deserción, e implementar las acciones que permitan dotar a los estudiantes que lo necesiten de las competencias necesarias para terminar exitosamente el pregrado e iniciar estudios de postgrado (Himmel 2003).

En atención a esto, investigadoras de la Universidad Santo Tomás (Délano, Errázuriz, y Fernández 2003) realizaron un estudio donde describen las principales características socioculturales, cognitivas (percepción de estrés y locus de control) y pedagógicas de una muestra de estudiantes de la misma universidad. Los resultados de su investigación indican que, en términos cognitivos, un grupo importante de estudiantes de la muestra presentan dificultades en la comprensión de las materias que el profesor imparte, así como problemas para organizar su tiempo con el fin de cumplir con los requerimientos académicos, lo que indicaría problemas con estrategias de aprendizaje, específicamente, en procesamiento metódico (Truffello y Pérez 1988). Por otro lado, un gran número de alumnos presenta altas expectativas de participación en la comunidad estudiantil, mismas que no son cumplidas. A la larga, ambos aspectos (cognitivo e integración social, respectivamente) pueden convertirse en factores de deserción.

Históricamente, los objetivos de la Educación Superior se han dirigido a promover habilidades y destrezas cognitivas a nivel individual. El trabajo apunta entonces a privilegiar el aprendizaje autónomo, por medio de la estimulación de la metacognición, motivando a los futuros graduados a seguir aprendiendo a lo largo de su vida, y disminuyendo además los factores cognitivos que afectan la deserción del sistema (Himmel 2003). Promover la metacognición es fundamental ya que hace referencia a las habilidades que tienen las personas para predecir sus desempeños en tareas variadas, así como para monitorear sus niveles actuales de dominio y comprensión (Brandsford et al. 2000).

A pesar de estos objetivos, hay evidencia de que los resultados obtenidos por los diferentes sistemas educativos tienden a la enseñanza en forma superficial y que se evalúa estableciendo estándares que pueden ser acordes a la enseñanza, pero que están por debajo del potencial del alumno (Beas 1994; Beas, Santa Cruz, Manterota y Carranza 2000; Alvarado, Sánchez y Uribe 2000; Romo, López, Tovar et al. 2004; VRA, Universidad Santo Tomás 2006). 
Esta situación llama urgentemente a buscar formas que faciliten el aprendizaje de destrezas cognitivas que fomenten el aprendizaje autónomo en los alumnos. La mejora en el rendimiento no es sólo producto del aumento en los contenidos ni de los cambios curriculares, sino también de la habilitación en destrezas intelectuales (Beas, Santa Cruz, Manterota y Carranza 2000).

En este contexto individual se hace indispensable establecer la relación entre las estrategias de aprendizaje utilizadas por los alumnos universitarios y el rendimiento académico con el fin de establecer un marco general acerca de cómo estas estrategias influyen en el proceso de enseñanza-aprendizaje a nivel de Educación Superior (Alvarado, Sánchez y Uribe 2000).

La importancia de entender las formas de procesamiento de la información radica en que, al comprender estos procesos, es posible mejorar el rendimiento académico, producto del incremento de la efectividad académica y del consiguiente aprendizaje significativo, que implica comprender, relacionar la nueva información con inclusores previos, interacción con los contenidos y relación con lo cotidiano (Alvarado, Sánchez y Uribe 2000; Brandsford et al. 2000; Romo, López, Tovar y López 2004).

Las distintas teorías existentes sobre estrategias de aprendizaje son modelos explicativos que han sido obtenidos de situaciones experimentales, y que pueden esclarecer relativamente el funcionamiento real de los procesos naturales del aprendizaje. Así las personas perciben y adquieren conocimientos, tienen ideas, piensan y actúan de manera distinta. Además tienen preferencias hacia una o más estrategias cognitivas que les ayudan a dar significado a una nueva información. Entonces, el término estilos de aprendizaje se refiere a esas estrategias preferidas que de manera más específica son formas de recopilar, interpretar, organizar y pensar sobre nueva información (Romo, López, Tovar y López 2004; Gázquez, Pérez, Ruiz et al. 2006).

A pesar de la cantidad y diversidad de acepciones que se pueden encontrar sobre estrategias de aprendizaje, es posible establecer que la mayoría de los autores coinciden en que se trata de cómo la mente procesa la información, del cómo es influenciada por las percepciones de cada individuo, todo con la finalidad de lograr aprender eficazmente (Romo, López, Tovar y López 2004).

Es posible encontrar distintas definiciones sobre estrategias de aprendizaje. Ronald Schmeck ha realizado investigaciones en el área de estrategias y tácticas de aprendizaje, fuera del laboratorio, formulando preguntas a los estudiantes sobre su modo cotidiano de estudiar y efectuando el análisis factorial de sus respuestas. Así, asume que cada uno de los grupos de tácticas revelados por el análisis factorial representa una estrategia y que el uso de tal estrategia representa un estilo (Schmeck 1980; Schmeck \& Lockard 1983; Schmeck \& Meier 1984, en Romo, López, Tovar y López 2004).

A través del modelo teórico de Schmeck (1980, en Romo, López, Tovar y López 2004) es posible identificar tres dimensiones de estrategias de aprendizaje: Procesamiento Profundo, Procesamiento Elaborativo y Procesamiento Superficial. Cada uno de ellos corresponde a un nivel de procesamiento de información, por lo cual se encuentra implícita la posibilidad de variación en el nivel de procesamiento, en función de la estimulación y requerimientos ambientales.

En la dimensión Procesamiento Profundo, el estudiante basa su aprendizaje en las asociaciones que le sugiere el concepto, más que al concepto mismo, es decir, el estudiante toma más tiempo en pensar que en repetir, pone atención a los rasgos semánticos 
y es capaz de clasificar, comparar, contrastar, analizar y sintetizar (Romo, López, Tovar y López 2004).

En el Procesamiento Elaborativo la información se hace personalmente más relevante, y el estudiante la elabora pensando en ejemplos personales y logra expresarse en sus propias palabras (Romo, López, Tovar y López 2004). Además, tiene un concepto de sí mismo más articulado, y asume que el aprendizaje implica adaptación, aplicación y desarrollo de mecanismos de almacenamiento (Schmeck y Meier 1984, en Truffello y Pérez 1988), lo que acarrea un aumento en la complejidad y riqueza del sí mismo.

El Procesamiento Superficial no es un estilo independiente, sino el extremo inferior en el continuo del procesamiento profundo. Todos los estudiantes procesan superficialmente, ya que sólo a través de esto (atendiendo a los símbolos) se puede llegar al procesamiento profundo (Truffello y Pérez 1988). El estudiante que utiliza este estilo prefiere asimilar la información tal como la recibe; define el aprendizaje creyendo que la verdad la aporta el sistema educativo, y que el deber del estudiante es guardar literalmente esta verdad en la memoria, en vez de reexpresarla, replantearla o repensarla. En su estudio atiende más a los aspectos fonológicos y estructurales de la información, invirtiendo mucho tiempo en repetir y memorizar información en su forma original (Romo, López, Tovar y López 2004).

Junto a las tres dimensiones, Schmeck (1980) plantea el Factor Estudio Metódico, referido a cómo el estudiante estudia: horarios, apuntes, y otros aspectos que están altamente relacionados con el procesamiento superficial, en cambio su relación con los procesamientos elaborativo y profundo no es significativa, es más, tiende a ser inversa (Romo, López, Tovar y López 2004).

La adaptación del inventario de Schmeck para Chile (Truffello y Pérez 1988) quedó formada por 55 enunciados distribuidos en cuatro factores.

El factor 1 corresponde al Procesamiento Elaborativo, semejante al descrito por Schmeck. El factor 2 corresponde al Estudio Metódico. El factor 3, denominado Procesamiento Profundo, está relacionado con el desarrollo del pensamiento crítico, atendiendo más al significado que a las características superficiales de la información. El factor 4 corresponde a la retención de Hechos, asociada a la clasificación de la información en categorías estrechas y precisas.

Por otra parte, numerosos estudios han aportado datos para sostener la hipótesis de la relación existente entre la imagen que el alumno tiene de sí mismo y la selección y/o utilización de estrategias de aprendizaje (Gázquez, Pérez, Ruiz et al. 2006).

En cuanto al concepto de autoestima, encontramos también diversidad de definiciones, aunque, si bien se puede afirmar que no son estrictamente excluyentes, tampoco se puede decir que sean complementarias. Hay consenso general en considerar la autoestima como un aspecto o dimensión del autoconcepto y como un modo de orientación hacia el sí mismo, haciendo referencia a través de ella, al valor que el individuo atribuye a su particular descripción de sí mismo (Gázquez, Pérez, Ruiz et al. 2006).

Algunos autores (Gázquez, Pérez, Ruiz et al. 2006) la definen como la clave del éxito o del fracaso para la autocomprensión y la comprensión de los demás, ya que afecta virtualmente en forma decisiva a todos los aspectos de nuestra experiencia. Al igual que se considera a la autoestima como un componente evaluativo/afectivo del autoconcepto, podemos describir que la autoestima tiene dos componentes: un sentimiento de capacidad personal y un sentimiento de valía personal. 
Múltiples trabajos ponen de manifiesto que cuando un sujeto se siente autocompetente se produce un aumento en su implicación activa en el proceso de aprendizaje (Miller, Behrens y Greene 1993; Núñez, González-Pienda, García, González-Pumariega, Roces, Álvarez y González 1998; Zimmerman, Bandura y Martínez-Pons 1992, en Gázquez, Pérez, Ruiz et al. 2006), siendo coincidentes algunos de ellos al poner de relieve la relación significativa entre el autoconcepto y la selección y/o utilización de determinadas estrategias de aprendizaje (Núñez y González-Pienda, 1994; Núñez et al. 1998, en Gázquez, Pérez, Ruiz et al. 2006). Además, apuntan hacia el mismo tipo de relación cuando se analiza el autoconcepto y la competencia o rendimiento académico, señalando que los estudiantes con altos sentimientos de autoeficacia académica se involucran más en tareas de aprendizaje, muestran alto nivel de esfuerzo, persisten más ante las dificultades, utilizan más eficazmente diversas estrategias de aprendizaje, tienen mayor capacidad de autorregular su propio aprendizaje, muestran mayor motivación intrínseca y se orientan más hacia la consecución de metas de aprendizaje que de ejecución (González y Tourón 1992; Marsh 1990, en Gázquez, Pérez, Ruiz et al. 2006).

Por el contrario, la carencia de estima propia aparece también sistemáticamente relacionada con ansiedad, depresión y sensación de fracaso, o la no aceptación de su propia imagen personal (Gila, Castro, Gómez y Toro, 2005, en Gázquez, Pérez, Ruiz et al. 2006), entre otros aspectos, que pueden inhibir el interés por el estudio y cuya modificación resulta extremadamente difícil en el aula, pero que pueden verse cambiados indirectamente al aumentar la autoestima del sujeto.

\section{OBJETIVOS:}

Objetivo general. Determinar la relación existente entre estrategias de aprendizaje, rendimiento académico y autoestima en los estudiantes de primer año UST, Talca, 2007.

\section{Objetivos específicos}

1. Describir las estrategias de aprendizaje y la autoestima en los estudiantes de primer año UST, Talca, 2007.

2. Determinar la relación entre estrategias de aprendizaje y rendimiento académico en los estudiantes de primer año UST, Talca, 2007.

3. Determinar la relación entre autoestima y rendimiento en los estudiantes de primer año UST, Talca, 2007.

4. Determinar la contribución relativa de las variables autoestima y estrategias de aprendizaje en el rendimiento académico de los estudiantes de primer año UST, Talca, 2007.

\section{METODO}

Diseño del estudio. Dada la naturaleza correlacional de los objetivos de la presente investigación, se llevó a cabo la implementación de un diseño no experimental, en base a una encuesta transversal aplicada a una muestra de estudiantes de la UST Talca.

El carácter correlacional de la investigación está dado porque el propósito principal del estudio es indagar la existencia de relación entre estrategias de aprendizaje y rendimiento 
académico. Por su parte, la caracterización como una encuesta transversal obedece al hecho de que la obtención de la información se llevó a cabo en base a la aplicación de un conjunto de instrumentos en un solo momento en el tiempo.

Universo. El universo de esta investigación está compuesto por el total de alumnos matriculados en la UST Talca con ingreso el año 2007.

Muestra. La muestra estuvo conformada por 371 estudiantes de ambos sexos, seleccionados en base a un muestreo no probabilístico, por accesibilidad. Este número de participantes permite satisfacer los requerimientos de potencia estadística y control del error tipo I, dados los procedimientos estadísticos multivariados que se emplearán en los análisis de los datos obtenidos (Stevens 2002).

$\mathrm{Al}$ analizar la edad de los participantes se observa que esta variaba de 18 a 45 años, con un promedio de 19.84 años $(D E=2.75)$. De estos, el 70,1\% $(n=260)$ correspondió a mujeres, mientras que el 29,9\% $(n=111)$ a hombres.

\section{Definición de variables}

Estrategias de aprendizaje. Modo/forma preferida o específica de procesar la información (estrategias cognitivas), que van desde la forma de recopilar, interpretar, hasta el organizar y pensar sobre una nueva información, todo con la finalidad de lograr aprender eficazmente (Romo, López, Tovar y López 2004; Gázquez, Pérez, Ruiz et al. 2006).

A través del modelo teórico de Schmeck (1980 citado en Romo, López, Tovar y López 2004) es posible identificar tres dimensiones de estilos de aprendizaje: procesamiento profundo, procesamiento elaborativo y procesamiento superficial.

En la dimensión procesamiento profundo, el estudiante basa su aprendizaje en las asociaciones que le sugiere el concepto más que al concepto mismo, es decir, el estudiante toma más tiempo en pensar que en repetir, pone atención a los rasgos semánticos y es capaz de clasificar, comparar, contrastar, analizar y sintetizar (Romo, López, Tovar y López 2004).

En el procesamiento elaborativo la información se hace personalmente más relevante, y el estudiante la elabora pensando en ejemplos personales y logra expresarse en sus propias palabras (Romo, López, Tovar y López 2004). Aquí el aprendizaje implica adaptación, aplicación y desarrollo de mecanismos de almacenamiento (Schmeck y Meier 1984 en Truffello y Pérez 1988).

El procesamiento superficial no es un estilo independiente, sino el extremo inferior en el continuo del procesamiento profundo. El estudiante que utiliza este estilo prefiere asimilar la información tal como la recibe; define el aprendizaje creyendo que la verdad la aporta el sistema educativo, y que el deber del estudiante es guardar literalmente esta verdad en la memoria, en vez de reexpresarla, replantearla o repensarla. En su estudio atiende más a los aspectos fonológicos y estructurales de la información (Romo, López, Tovar y López 2004).

Junto a las tres dimensiones, Schmeck (1980) plantea el factor estudio metódico, referido a cómo el estudiante estudia: horarios, apuntes, y otros aspectos (Romo, López, Tovar y López 2004).

Las estrategias de aprendizaje se midieron a través del Inventario de Estrategias de Aprendizaje de Ronald Schmeck. 
Autoestima. Se define como "la cognición que el individuo tiene conscientemente sobre sí mismo; influyen todos los atributos, rasgos y características de personalidad que estructuran y se incluyen en lo que el individuo considera como su yo. Podríamos considerarlo como equivalente al concepto de sí mismo o autoimagen" (Brinkmann, Sègure y Solar 1989: 13).

Si bien la autoestima se va formando a lo largo de toda la vida, en la adolescencia la percepción y valoración que tiene el joven de sí mismo toma un auge importante para lograr una de las tareas más esenciales que es el logro de la identidad; al igual que esta identidad, la autoestima consta de varios elementos, por lo que podemos decir que es multidimensional, ya que reúne aspectos de tipo social, moral, físico, cognitivo y sexual (Papalia, Olds, Feldman, Pineda, et al. 2005).

Para evaluar la autoestima se utilizó el Inventario de Autoestima de Coopersmith forma $A$, versión adaptada para adolescentes chilenos (Brinkmann, Sègure y Solar 1989).

Rendimiento Académico. Grado o nivel de logros alcanzados por los estudiantes de la Universidad Santo Tomás (UST), según las notas finales obtenidas durante el primer semestre, arrojado por el sistema CLAS como promedio ponderado de todas las asignaturas cursadas durante el primer semestre 2007.

Operacionalmente esta variable se expresará en nota de 1.0 a 7.0, considerando sólo un decimal.

Como un antecedente adicional, se consignó el número de asignaturas reprobadas en el primer semestre, arrojado por el sistema CLAS. Operacional esta variable se expresa en numerales.

Instrumentos. La obtención de los datos se llevó a cabo en base a un cuestionario de antecedentes sociodemográficos y las escalas que se describen a continuación.

Inventario de Estrategias de Aprendizaje de Ronald Schmeck. La adaptación del inventario para Chile (Truffello y Pérez 1988) está formada por 55 enunciados distribuidos en cuatro subescalas: a) procesamiento profundo, b) procesamiento elaborativo, c) retención de hechos y d) estudio metódico. El Inventario permite ubicar a los alumnos en el estilo de aprendizaje predominante.

En cuanto a la consistencia interna de las subescalas obtenidas en la presente investigación, estas correspondieron a un alfa de 0.76 para procesamiento profundo, 0.59 para procesamiento elaborativo, 0.60 para retención de hechos y 0.74 para estudio metódico.

Inventario de autoestima de Coopersmith forma A. Corresponde a la validación llevada a cabo por Brinkmann, Sègure y Solar (1989). Es un instrumento de autorreporte de 58 ítemes frente a los cuales el participante debe indicar si la afirmación que se le presenta es "Igual que yo" o "Distinto a mî". El inventario entrega un puntaje de autoestima general, social, familiar (hogar y padres) y académica, además consta de una escala de mentira.

En cuanto a la consistencia interna de las subescalas obtenidas en la presente investigación, estas correspondieron a un alfa de 0.77 para autoestima general, 0.39 para autoestima social, 0.65 para autoestima familiar y 0.54 para autoestima académica. Debido a la baja confiabilidad reportada por la subescala de autoestima social, en los análisis que se informan a continuación no se incluyeron los datos entregados por esta subescala. 
Recolección de información. La aplicación de los instrumentos se llevó a cabo por un grupo de estudiantes de psicología que fueron capacitados para esta actividad, a modo de estandarizar la aplicación, la cual se realizó durante la jornada de clases.

Los datos recogidos a través de la encuesta fueron vaciados en una base de datos de programa computacional.

Análisis estadísticos. Una vez finalizada la obtención de los datos, se procedió a construir la base de datos en una planilla electrónica Excel. Posteriormente, los análisis estadísticos se llevaron a cabo en base a los programas estadísticos SPSS 13.0.

Una vez importados los datos y, como primera actividad, se llevó a cabo la inspección de los mismos, a objeto de detectar posibles valores fuera de rango.

A continuación, se procedió a llevar a cabo los análisis estadísticos pertinentes a los objetivos de esta investigación.

Se llevaron a cabo análisis exploratorio/descriptivo de los datos a objeto de identificar las características distribucionales de las diversas variables bajo estudio, presencia de valores atípicos, determinación de estadísticos de tendencia central y variabilidad.

Junto a lo anterior, se procedió a determinar la confiabilidad de las escalas empleadas en el estudio. Para ello se analizó su consistencia interna en base al empleo del coeficiente alfa de Cronbach.

A objeto de clasificar a los participantes en términos de sus estrategias de aprendizaje, se realizó un Análisis de Conglomerados. En cuanto a los análisis bivariados empleados, se efectuó la aplicación de los estadísticos Wilcoxon-Mann-Whitney y coeficiente de correlación producto-momento de Pearson. Finalmente, se realizó un Análisis de Regresión Lineal Múltiple.

Al momento de llevar a cabo el empleo de las diferentes técnicas estadísticas, se tuvo especial cuidado en evaluar el adecuado cumplimiento de los supuestos de cada una de ellas, de modo de garantizar una correcta interpretación de sus resultados. Como criterio para determinar la significación estadística de los resultados se empleó el valor $\alpha=.05$

\section{RESULTADOS}

Descripción de los estudiantes en base a sus estrategias de aprendizaje y autoestima. Un primer objetivo del presente estudio fue describir los estilos de aprendizaje y la autoestima evidenciados por los estudiantes encuestados.

En la tabla 1 se presentan los resultados obtenidos por la muestra de estudiantes en cada uno de los estilos de aprendizaje evaluados por el Inventario de Schmeck.

Como se aprecia en la tabla 1 , los promedios alcanzados en las escalas de procesamiento profundo, procesamiento elaborativo y estudio metódico sitúan a los estudiantes encuestados dentro del rango Normal en cada una de ellas. La única diferencia a este patrón corresponde al promedio obtenido en la escala de retención de hechos, en los que los estudiantes se sitúan en la categoría Baja.

En cuanto a los resultados obtenidos en las distintas escalas del cuestionario de autoestima de Coopersmith, estos se presentan en la tabla 2. 


\section{Tabla 1}

Promedio y desviación estándar en las subescalas del Inventario de Schmeck $(N=371)$

\begin{tabular}{|l|c|c|}
\hline & $M$ & $D E$ \\
\hline Profundo & 10.43 & 3.49 \\
\hline Elaborativo & 5.79 & 1.77 \\
\hline Metódico & 8.46 & 3.14 \\
\hline Hechos & 7.38 & 2.82 \\
\hline
\end{tabular}

Tabla 2

Promedio y desviación estándar en las subescalas del Cuestionario de Autoestima de Coopersmith $(N=371)$

\begin{tabular}{|l|c|c|}
\hline & $M$ & $D E$ \\
\hline General & 41.54 & 7.54 \\
\hline Académica & 10.70 & 3.28 \\
\hline Famil Familiar & 10.58 & 3.78 \\
\hline
\end{tabular}

$\mathrm{Al}$ analizar los resultados presentados en la tabla 2, se concluye que los estudiantes encuestados exhiben puntajes en las escalas de autoestima analizadas en torno a los valores normales.

Clasificación de los participantes en base a sus estrategias de aprendizaje. Como paso previo a la evaluación de la Hipótesis 1, se procedió a clasificar el conjunto de participantes del estudio en términos de sus estrategias de aprendizaje. Para ello se sometieron a un Análisis de Conglomerados los puntajes obtenidos en las cuatro escalas del Inventario de Estrategia de Aprendizaje de Schmeck.

La implementación de esta estrategia analítica contempló el uso conjunto de dos algoritmos de conglomeración. En una primera etapa, se sometió el conjunto de datos a un Análisis de Conglomerados Jerárquico Aglomerativo, mientras que en la segunda etapa se analizaron los mismos datos en base a un Análisis de Conglomerados No Jerárquico.

La razón de emplear ambos métodos obedece a lograr un adecuado balance entre las ventajas y desventajas de ambas estrategias analíticas. De este modo, los resultados del Análisis de Conglomerados Jerárquico permitieron identificar el número de conglomerados que de mejor forma logra clasificar al conjunto de participantes del estudio; mientras que el Análisis de Conglomerados No Jerárquico permitió una asignación más óptima de los participantes en los distintos conglomerados identificados previamente, a objeto de lograr una alta homogeneidad intraconglomerados y alta heterogeneidad entre los conglomerados. 
La realización del Análisis de Conglomerados Jerárquico se llevó a cabo empleando el método de Ward, utilizando como medida de distancia entre los datos la distancia euclídea al cuadrado.

Al momento de calcular la matriz de distancias, se procedió previamente a la estandarización de las variables, a objeto de evitar la influencia indebida en la solución de conglomerados final de aquellas variables que evidenciaran una mayor variabilidad.

Los resultados del análisis anterior llevaron a identificar como mejor solución de conglomerados una solución de dos conglomerados (véase figura 1).

\section{Figura 1}

Dendrograma obtenido mediante el análisis de conglomerado jerárquico (método de Ward)

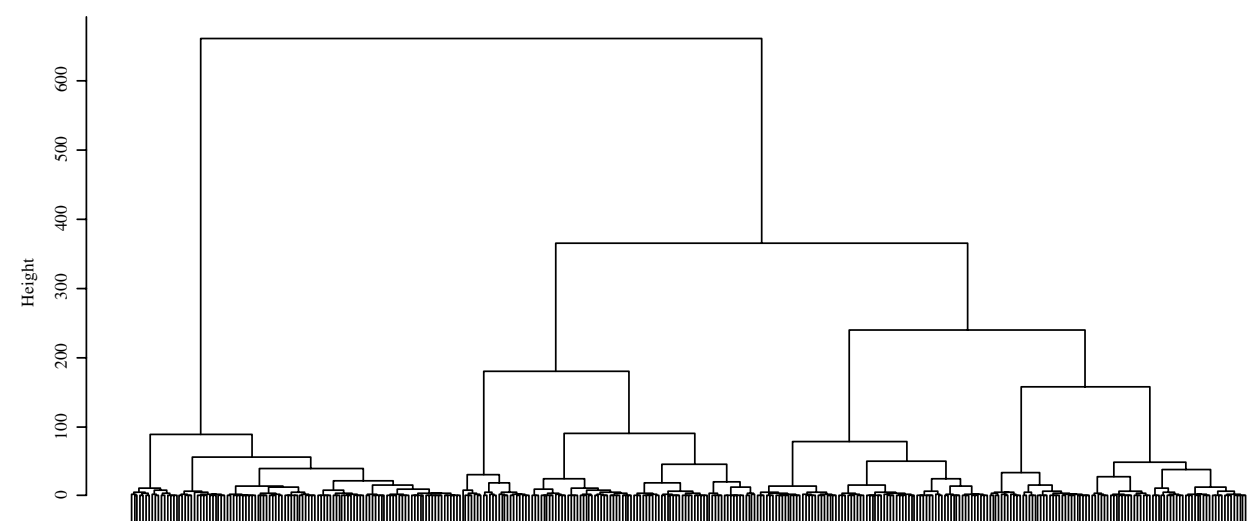

La tabla 3 presenta los estadísticos descriptivos en las cuatro subescalas del Inventario de Schmeck (variables sin estandarizar) para cada uno de los conglomerados identificados.

Tabla 3

Comparación de resultados en las subescalas del Inventario de Schmeck para la solución de dos conglomerados (método de Ward)

\begin{tabular}{|l|c|c|c|c|}
\hline \multirow{2}{*}{} & \multicolumn{2}{|c|}{ Conglomerado $1(N=110)$} & \multicolumn{2}{c|}{ Conglomerado 2 $(N=261)$} \\
\cline { 2 - 5 } & $M$ & $D E$ & $M$ & $D E$ \\
\hline Profundo & 13.25 & 1.88 & 9.23 & 3.32 \\
\hline Elaborativo & 6.56 & 1.35 & 5.46 & 1.83 \\
\hline Metódico & 10.91 & 1.95 & 7.43 & 2.98 \\
\hline Hechos & 5.09 & 1.95 & 8.35 & 2.56 \\
\hline
\end{tabular}


Como se observa en la tabla 3, el primer conglomerado se caracteriza por exhibir puntajes más altos en las subescalas "Profundo", "Elaborativo" y "Metódico", mientras que el segundo conglomerado presenta puntajes más altos en la subescala "Hechos". De este modo, se observa una contraposición entre aquellos estudiantes que emplean estrategias de aprendizaje más complejas (conglomerado 1) en comparación a aquellos estudiantes que emplean estrategias de aprendizaje más simples (conglomerado 2).

Con el propósito de optimizar la asignación de los participantes a los dos conglomerados identificados, se procedió a complementar los resultados obtenidos con el método de Ward, mediante la aplicación de un Análisis de Conglomerados No Jerárquico en base al método de K-Medias.

Al igual que en el caso del método de Ward, el análisis mediante K-Medias se llevó a cabo sobre los puntajes estandarizados de las cuatro escalas del Inventario de Schmeck. Al momento de efectuarse la aplicación de este método, se procedió a asignar como centros iniciales para la construcción de los conglomerados, los promedios de cada uno de los dos conglomerados en las cuatro escalas del Inventario de Schmeck obtenidos por el método de Ward.

La tabla 4 presenta los estadísticos descriptivos en las cuatro subescalas del Inventario de Schmeck (variables sin estandarizar) para cada uno de los conglomerados obtenidos mediante K-Medias.

Al comparar los promedios que presenta la tabla 4 para los dos conglomerados analizados, se puede concluir que los resultados obtenidos mediante K-medias son bastante similares a aquellos obtenidos mediante el método de Ward.

Es así como se observa que los estudiantes que integran el primer conglomerado se caracterizan por presentar puntajes más altos en las subescalas "Profundo", "Elaborativo" y "Metódico", mientras que los estudiantes que integran el segundo conglomerado exhiben puntajes más altos en la subescala "Hechos". Una vez más, estos resultados estarían contraponiendo a aquellos estudiantes que emplean estrategias de aprendizaje más complejas (conglomerado 1) con aquellos estudiantes que emplean estrategias de aprendizaje más simples (conglomerado 2).

\section{Tabla 4}

Comparación de resultados en las subescalas del Inventario de Schmeck para la solución de dos conglomerados (método de K-Medias)

\begin{tabular}{|l|c|c|c|c|}
\hline \multirow{2}{*}{} & \multicolumn{2}{|c|}{ Conglomerado l $(N=193)$} & \multicolumn{2}{c|}{ Conglomerado 2 (N=178) } \\
\cline { 2 - 5 } & $M$ & $D E$ & $M$ & $D E$ \\
\hline Profundo & 12.42 & 2.28 & 8.26 & 3.29 \\
\hline Elaborativo & 6.64 & 1.31 & 4.87 & 1.75 \\
\hline Metódico & 9.69 & 2.85 & 7.12 & 2.89 \\
\hline Hechos & 5.63 & 2.14 & 9.29 & 2.16 \\
\hline
\end{tabular}


Rendimiento académico y su relación con estrategias de aprendizaje y autoestima. Una vez clasificados los participantes en términos de sus estrategias de aprendizaje, el paso siguiente en este estudio fue analizar si existían diferencias significativas en cuanto a su rendimiento académico. Para ello se comparó el promedio de notas y el número de asignaturas reprobadas entre los participantes de ambos conglomerados.

Al comparar las notas, se observa que el promedio de los estudiantes con estrategias complejas correspondió a $4.70(D E=0,63)$, mientras que para los estudiantes con estrategias más simples este promedio fue de $4.44(D E=0.58)$. El estadístico t de student para muestras independientes en base a un contraste unilateral proporcionó un resultado de $t$ (369) $=4.13, p<0.001$. La diferencia en los promedios correspondió a 0.26 (95\% IC: 0.14 a 0.38 ), lo que permite concluir que los estudiantes del primer grupo obtienen un rendimiento académico significativamente más alto que los estudiantes del segundo grupo. En cuanto al tamaño del efecto, este correspondió a $g=0.10$ (Kline 2005) que, de acuerdo a los criterios propuestos por Cohen (1992) para la prueba $t$, representa un efecto leve.

Por su parte, cuando se compara el número de asignaturas reprobadas por los participantes de cada conglomerado se tienen los resultados que presentan en la tabla 5.

Como se observa en la tabla 5, los participantes del conglomerado estrategias más complejas tienden a mostrar un menor número de asignaturas reprobadas en comparación a los participantes de estrategías más simples. Es así como se observa que del total de participantes de estrategias complejas, el $42,97 \%$ ha reprobado alguna asignatura, mientras los estudiantes de estrategias más simples este porcentaje asciende al 65,17\%.

Para determinar si existían diferencias en el número de repitencias que exhibían los participantes de ambos conglomerados, se empleó el estadístico de Wilcoxon-Mann-Whitney. El resultado obtenido correspondió a $Z=4,31, p<0,001$, lo cual permite concluir que existen diferencias significativas entre ambos grupos, siendo los participantes que utilizan estrategias de aprendizaje más complejas quienes presentan menores reprobaciones.

Una segunda línea de análisis del presente estudio correspondió a examinar la existencia de relación entre la autoestima de los estudiantes y su rendimiento académico.

\section{Tabla 5}

Comparación de resultados en el número de asignaturas reprobadas para la solución de dos conglomerados (método de K-Medias)

\begin{tabular}{|c|c|c|c|c|}
\hline \multirow{2}{*}{$\begin{array}{c}N^{o} \text { de } \\
\text { reprobaciones }\end{array}$} & \multicolumn{2}{|c|}{ Conglomerado 1 } & \multicolumn{2}{c|}{ Conglomerado 2 } \\
\cline { 2 - 5 } & $N$ & $\%$ & $N$ & $\%$ \\
\hline 0 & 112 & 58,03 & 62 & 34,83 \\
\hline 1 & 31 & 16,06 & 41 & 23,03 \\
\hline 2 & 25 & 12,95 & 34 & 19,10 \\
\hline 3 & 12 & 6,22 & 22 & 12,36 \\
\hline 4 & 11 & 5,70 & 16 & 8,99 \\
\hline 5 & 1 & 0,52 & 2 & 1,12 \\
\hline 6 & 1 & 0,52 & 1 & 0,56 \\
\hline Total & 193 & 100,0 & 178 & 100,0 \\
\hline
\end{tabular}


Para evaluar la hipótesis que planteaba que los estudiantes que exhibieran mayores niveles de autoestima exhibirían un mayor rendimiento académico se empleó el coeficiente de correlación producto-momento de Pearson (véase tabla 6).

\section{Tabla 6}

Matriz de correlaciones producto-momento de Pearson entre notas y subescalas del Cuestionario de Autoestima de Coopersmith $(N=371)$

\begin{tabular}{|l|c|c|c|c|}
\cline { 2 - 5 } \multicolumn{1}{c|}{} & 1 & 2 & 3 & 4 \\
\hline 1: Notas & - & & & \\
\hline 2: A. General & $.17^{* * *}$ & $(.77)^{\mathrm{a}}$ & & \\
\hline 3: A. Académica & $.36^{* * *}$ & $.54 * * *$ & $(.54)$ & \\
\hline 4: A. Familiar & $.17^{* * *}$ & $.46^{* * *}$ & $.35^{* * *}$ & $(.65)$ \\
\hline
\end{tabular}

a: entre paréntesis valores alfa de Cronbach. $*: \mathrm{p}<.05 ; * *: \mathrm{p}<.01 ; * * *: \mathrm{p}<.001$.

Del análisis de la tabla 6 se concluye que los estudiantes que exhiben un mayor rendimiento académico tienden a caracterizarse por presentar mayores niveles de autoestima general $(r(369)=.17)$, mayores niveles de autoestima académica $(r(369)=.36) \mathrm{y}$ mayores niveles de autoestima familiar $(r(369)=.17)$.

Finalmente, para analizar el efecto conjunto de los estilos de aprendizaje y las dimensiones de autoestima sobre el rendimiento académico, se llevó a cabo un análisis de regresión lineal múltiple.

Al considerar la capacidad explicativa global del modelo, se observa un $R^{2}=.142$ $\left(R^{2}\right.$ ajustado de .133$), F(4,366)=15.179, p<.001$, lo cual indica que aproximadamente el $14 \%$ de la variabilidad de los promedios de notas son predichos por el conjunto de predictores. En cuanto a los valores de los coeficientes para el modelo estos se presentan en la tabla 7.

\section{Tabla 7}

Coeficientes, niveles de significación y correlaciones semiparciales al cuadrado para los resultados de la regresión lineal múltiple $(N=371)$

\begin{tabular}{|l|c|c|c|c|}
\hline & $B$ & $E E$ & $\beta$ & $s r^{2}$ \\
\hline Intercepto & $3.96^{* * *}$ & .171 & & \\
\hline E. Aprendizaje & $.125^{*}$ & .065 & .101 & .009 \\
\hline General & -.006 & .005 & -.073 & .003 \\
\hline A. Académica & $.064^{* * *}$ & .011 & .341 & .077 \\
\hline Familiar & .010 & .009 & .064 & .003 \\
\hline
\end{tabular}

$*: \mathrm{p}<0.05 ; * *: \mathrm{p}<0.01 ; * * *: \mathrm{p}<0.001$. 
Del análisis de la tabla 7 se puede concluir que sólo dos de los cuatro predictores resultan ser estadísticamente significativos en su contribución a la explicación de los promedios de notas. De estos dos predictores, el que presenta una mayor relación con la variable criterio corresponde a la dimensión de autoestima académica, siendo los estudiantes que exhiben mayores niveles de esta variable quienes presentan mayores calificaciones $\left(\beta=.341, s r^{2}=.077\right)$. El segundo predictor corresponde al estilo de aprendizaje, siendo los estudiantes que emplean estrategias de aprendizaje más complejas quienes obtienen mayores calificaciones $\left(\beta=.101, s r^{2}=.009\right)$. Sin relación estadísticamente significativa aparecen la autoestima general $(\mathrm{p}>.05)$ y la autoestima familiar $(\mathrm{p}>.05)$.

\section{CONCLUSIONES}

Los resultados observados indican que los alumnos ingreso 2007 de la Universidad Santo Tomás sede Talca exhiben puntajes de autoestima general, académica y familiar dentro de los rangos normales (según estandarización Brinkmann, Sègure y Solar 1989) Lo que es congruente con lo planteado por González y Uribe (2002) en relación al grupo de estudiantes que ingresa a la educación superior ya no es una elite, sino un grupo, más grande, de características "normales".

Las estrategias de aprendizaje de los alumnos de primer año UST Talca se distribuyen en dos grandes conglomerados. Uno de los conglomerados, que reúne el 52\% de los alumnos $(\mathrm{N}=193)$, se caracteriza por el uso de estrategias de aprendizaje más complejas (profundas, elaborativas y metódicas).

Descriptivamente este grupo se caracteriza por usar principalmente, pero no exclusivamente, el lenguaje y sus significados como elementos ordenadores, siendo las características semánticas de la información las claves centrales a la hora de codificar y registrar. Esto permite que la información sea integrada en una red de inclusores previos, que facilitan su recuperación, su generalización y transferencia a otros contextos y contenidos. De este modo, la información puede ser clasificada y jerarquizada, facilitando la formación de conceptos. A su vez, estos alumnos conocen cómo aprenden, es decir, han desarrollado habilidades de metacognición, implementando estrategias operativas que les permiten un aprendizaje eficiente, según sus propias destrezas de aprendizajes. Este estudiante es proactivo frente al desafío de aprender. Desarrolla un andamiaje con esquemas, resúmenes, mapas conceptuales, y busca sus propios ejemplos para poder entender la información.

Además, se caracteriza por poner en práctica una disciplina o metódica de trabajo, es decir, establece una rutina de horarios, cumple con tareas y requerimientos solicitados y ha desarrollado una organización o rutina de estudio.

El otro conglomerado, que reúne el $48 \%$ de los alumnos $(\mathrm{N}=178)$, utiliza principalmente estrategias más simples o superficiales ("hechos") para su aprendizaje. Se caracterizan por aprender repitiendo (repaso repetitivo), donde la información se codifica por sus características superficiales y no semánticas. Prima un registro de la información de modo aislado, desarticulado de los conocimientos anteriores, la comprensión e integración de los nuevos contenidos no es una estrategia frecuente. Es un sujeto más bien pasivo frente a su proceso de aprendizaje, no busca nueva información que enriquezca, complemente o le ayude a entender los nuevos contenidos. 
En cuanto a la relación Estrategias de Aprendizaje, Autoestima y Rendimiento Académico, se observó que los estudiantes universitarios que utilizan estrategias de aprendizaje más complejas presentan significativamente mejor rendimiento académico, y significativamente menos reprobaciones que el grupo de estudiantes que utiliza estrategias de aprendizaje más simples.

Los estudiantes que exhiben un mayor rendimiento académico tienden a caracterizarse por presentar mayores niveles de autoestima general, mayores niveles de autoestima académica y mayores niveles de autoestima familiar.

Ahora bien, al evaluar el nivel predictivo de las variables de nuestro modelo se observa que la autoestima académica y las estrategias de aprendizaje son predictores estadísticamente significativos, siendo la primera la que presenta la mayor relación con el rendimiento académico.

De este modo, podemos decir que una buena autoestima académica y la utilización de estrategias de aprendizaje complejas predicen un buen rendimiento, a la vez que se retroalimentan entre sí; lo contrario también es posible, utilización de estrategias de aprendizaje simples predicen menor rendimiento, y menos autoestima académica. Esto genera un círculo vicioso, que deja vulnerable a un grupo de estudiantes, transformándolos en posibles desertores de la Educación Superior.

\section{DISCUSION}

De este modo, las estrategias de aprendizaje utilizadas, el rendimiento académico logrado y la autoestima académica se configuran como factores relevantes en la permanencia o deserción en la Educación Superior. Surgen así muchas interrogantes: ¿Cómo intervenir en este círculo? ¿Cuáles son las áreas en las que hay que intervenir? ¿Cuál es la relación de estos factores con la deserción en Educación Superior?

Son escasas las investigaciones acerca de los factores predictores de deserción y retención universitaria (González y Uribe 2002; Himmel 2002), sin embargo, se han desarrollado distintos enfoques de análisis del fenómeno. Braxton et al. 1977 (citado por Himmel 2002) distingue a lo menos 5 enfoques de análisis: psicológico, económico, sociológico, organizacional y finalmente un enfoque interaccional que integra la influencia de todos estos factores. Como se puede apreciar, los factores que interfieren en la deserción en la educación superior van más allá de las variables psicológicas estudiadas, y se hace evidente la necesidad de desarrollar investigación que involucre todas las dimensiones.

Sin embargo, es posible plantear algunas intervenciones desde la perspectiva de las estrategias de aprendizaje y la autoestima académica que permitan contribuir a potenciar las habilidades de los estudiantes.

Algunas de ellas pueden ser:

1. Promover prácticas docentes que estimulen la utilización de estrategias de aprendizaje elaborativas y profundas. Es decir, que en el proceso de enseñanza-aprendizaje se parta de los inclusores previos que traen los alumnos, para que los nuevos significados puedan ser enlazados con los preexistentes. Para ello debieran promoverse, por ejemplo, ejercicios de asociación, generalización y comparación de contenidos.

2. Realizar evaluaciones que busquen no sólo el reconocimiento de los contenidos, sino su comprensión, su diferenciación con otros, su aplicación y transferencia a 
otras áreas. En este sentido, es necesaria la utilización de pruebas con preguntas de desarrollo, la evaluación y el trabajo de argumentaciones y contraargumentaciones, la utilización de la modalidad de ensayo, entre otros.

3. Desarrollar en los alumnos la metacognición, es decir, la capacidad de conocer el modo como aprende cada uno. En este sentido debiera promoverse la identificación y/o toma de conciencia de las propias estrategias, como una habilidad básica para hacerse activo frente al propio aprendizaje.

4. Utilizar aquellas asignaturas del currículum que apuntan a la formación general, para fomentar método y disciplina de estudio.

5. Lo anterior pudiese dar paso a la implementación de un curso propedéutico ${ }^{l}$, que permita a los alumnos tener una estimulación adicional, que promueva el logro de estrategias más complejas, a la vez que refuerce habilidades interpersonales que faciliten su proceso de integración al nuevo sistema, que requerirá nuevas exigencias cognitivas. Dentro de esta mirada, cobra sentido la implementación de currículos más generalistas en los dos primeros años, donde se prepara a los alumnos para un segundo proceso de formación más autónomo, que implica la utilización de estrategias de elaboración de información más complejas, para los años de formación más específicos de cada profesión y los estudios de postgrado.

6. Está descrito que la integración social de los alumnos al sistema de Educación Superior constituye un factor preponderante para la inserción o la deserción al sistema. Se propone entonces utilizar las asignaturas de formación general para trabajar la integración y el sentido de pertenencia de los alumnos, disminuyendo la deserción. Por otro lado, y también coherente con la bibliografía, se propone el perfeccionamiento de los beneficios socioeconómicos que pueden ofrecer las instituciones universitarias (becas, ayudas financieras, servicio de salud, entre otros). Todo aquello que facilite y promueva la integración de los jóvenes al sistema universitario, de modo que ésta no se convierta en un obstáculo para su adaptación. Se sugieren aquí actividades de recepción y acogida, períodos de inducción a la operatoria universitaria general, entre otras.

Finalmente, es necesario recalcar que se hace imperativo integrar al modelo explicativo/comprensivo de la deserción universitaria en el Chile actual, no sólo variables psicológicas, sino que variables económicas, sociológicas, psicosociales, tales como el proceso de integración al sistema social universitario, el grado de satisfacción con la institución elegida, recursos económicos disponibles, acceso a redes, entre otras, a modo de entender los fenómenos educativos dentro de la multifactorialidad y multicausalidad que caracteriza a los fenómenos humanos.

1 Esto ya se ha implementado en algunas universidades bajo la modalidad de Bachillerato. 


\section{BIBLIOGRAFIA}

Alvarado H., I. Sánchez y M. Uribe (2000). Correspondencia entre estrategias de aprendizaje y rendimiento académico en estudiantes universitarios, Boletín de Investigación Educacional Facultad de Educación, Pontificia Universidad Católica de Chile, 15: 70- 88.

Beas Franco, J. (1994). ¿Qué es el pensamiento de buena calidad? Estado de avance de la discusión, Pensamiento Educativo 15: 13-28.

Beas, J., J. Santa Cruz, M. Manterota et al. (2000). Proposición de un diseño para evaluar el aprendizaje profundo. Boletín de Investigación Educacional Facultad de Educación, Pontificia Universidad Católica de Chile 15: 60-69.

Brandsford, J. et al. (editors). (2000). How people learn. Brain, mind, experience and school. USA: National Academy Press.

Brinkmann, H., T. Sègure y M. I. Solar (1989). Adaptación y estandarización del Inventario de Autoestima de Coopersmith, Revista Chilena de Psicología 10 (1): 73-87.

Canales, A. y D. De los Ríos (2007). Factores explicativos de la deserción universitaria. Informe final proyecto Consejo Superior de Educación, CICES - Universidad de Santiago de Chile, Estudios y documentos del Consejo Superior de Educación. [On line] Disponible en la World Wide Web: <www.cse.cl/public/Secciones/seccionestudios/estudios_y_documentos_de_la_SecTec. aspx $>$ Obtenido el 23/01/08

Délano, P., A. Errázuriz y B. Fernández (2003). Diagnóstico sociocultural, pedagógico y cognitivo (estilos de afrontamiento y locus de control) de los estudiantes de la Universidad Santo Tomás. Informe Final [On line] Disponible en la World Wide Web: <http://www.ust.cl/investigacion/ paginas/proyectos_investigacion.html> Obtenido el 13/03/07

Gázquez, J., M. Pérez, M. I. Ruiz et al. (2006). Estrategias de aprendizaje en estudiantes de enseñanza secundaria obligatoria y su relación con la autoestima. International Journal of Psychology and Psychological Therapy 6 (1): 51-62.

González, L. E. y D. Uribe (2002). Estimaciones sobre la "repitencia” y deserción en la Educación Superior chilena. Consideraciones sobre sus implicaciones. Revista Calidad de la Educación Superior $17,2^{\circ}$ Semestre. [On line] Disponible en la World Wide Web: <www.cse.cl/public/ Secciones/seccionpublicaciones/publicaciones_revista_calidad_detalle.aspx?idPublicacion=35> Obtenido el 18/01/08.

Himmel, E. (2002). Modelos de análisis de la deserción estudiantil en la Educación Superior. Revista Calidad de la Educación Superior 17, $2^{\circ}$ Semestre. [On line] Disponible en la World Wide Web: <www.cse.cl/public/Secciones/seccionpublicaciones/publicaciones_revista_calidad_detalle.aspx?idPublicacion=35> Obtenido el 18/01/08.

Himmel, E. (2003). Evaluación de aprendizajes en la Educación Superior: Una reflexión necesaria. Pensamiento Educativo 33: 199-211.

Lemaitre, M. J. (2003). Aseguramiento de la calidad en la Educación Superior: Opciones y modelos. Pensamiento Educativo 33: 212-229.

Montes, H. (2002). La transición de la Educación Media a la Educación Superior.

Revista Calidad de la Educación Superior 17, $2^{\circ}$ Semestre. [On line] Disponible en la World Wide Web: <www.cse.cl/public/Secciones/seccionpublicaciones/publicaciones_revista_calidad_detalle.aspx?idPublicacion=35> Obtenido el 18/01/08.

Papalia, D., R. Wenks y R. Dusckih (2004). Desarrollo Humano, Santa Fe, Bogotá: Mc GrawHill.

Pérez, F. e I. Truffello (1998). Diseño y evaluación de actividades instruccionales conducentes a las estrategias de aprendizaje elaborativa y profunda, Revista Enfoques Educacionales 1, $\mathrm{N}^{\circ}$ 1. [On line] Disponible en la World Wide Web: < http://www.facso.uchile.cl/publicaciones/ enfoques/01/docs/enfoques_01_1998.pdf > Obtenido el 26/02/07.

Prewitt Diaz, J. O. (1984). A cross-cultural study of the reliability of the Coopersmith Self Esteem Inventory, Educational and Psychological Measurement 44: 575-580. 
Romo Aliste, M. E., D. López Real, J. Tovar Martínez et al. (2004). Estilos de aprendizaje de estudiantes de nutrición y dietética, Facultad de Medicina, Universidad de Chile, Revista Praxis 5. [On line] Disponible en la World Wide Web: <www.revistapraxis.cl/ediciones/numero5/ romo_praxis5.html> Obtenido el 22/02/07.

Stevens, J. (2002). Applied Multivariate Statistics for the Social Sciences. Hillsdale, N.J.: L. Erlbaum.

Truffello, I. y F. Pérez (1988). Adaptación en Chile del "Inventory of Learning Processes" de Ronald Schmeck. Boletín de Investigación 6 (1- 2): 109-120.

Vicerrectoría Académica Universidad Santo Tomás. (2006). Informe Examinación Nacional $1^{\text {er }}$ Semestre 2006. Memorando de circulación interna, 20 de diciembre de 2006. 